Arch. hist. jap. Vol. 15, n. 4 (November 1958). S. $565-585$.

Anat. Inst. d. Med. Fak., Univ. Okayama (Vorstand: Prof. M. SEKI).

\title{
Bau des Ligamentum teres uteri in verschiedenem Lebensalter.
}

\author{
種々の生令に於ける子宮円索の構造.
}

Yasumitsu TAKEBAYASHI 竹林康光.

(Eingegangen am 30. September 1958.)

Über den mikroskopischen Bau des Ligamentum teres uteri liegen nur wenige Angaben vor. KOELLIKER wies in seinem Handbuch 1902 auf die Neubildung und Hyperplasie der glatten Muskelfasern im Ligamentum in der Schwangerschaftszeit hin. Später untersuchte KEIFFER (1925) das Ligamentum von verschiedenem Lebensalter und bemerkte die größere Zahl von elastischen Fasern im Ranteil des Bandes. MIYAZAKI (1931) konstațierte die Vermehrung der Dicke, Zahl und Schlängelung der elastischen Fasern im Ligamentum mit zunehmendem Alter und Wiederholung der Geburt. In vorliegender Arbeit hat der Verfasser die faserigen Elemente des Ligementums, nämlich Kollagen-, elastische, retikuläre und glatte Muskelfasern, von Föten und nicht- und bereits gebärenden Weibern von verschiedenem Alter untersucht.

\section{Mateial und Methode.}

Es wurden Untersuchungen am Material aus zwei Föten von 7 Monaten, einem Fötus von 9 Monaten, vier Föten von 10 Monaten, 32 Weibern von 2 Lebensdezennien, 15 Weibern von 3 Lebensdezennien, 23 Weibern von 4 Lebensdezennien, 3 Weibern von 5 Lebensdezennien und 8 Weibern von noch höcherem Alter vorgenommen. Man entnahm immer ein etwa $3 \mathrm{~mm}$ langes Ligamentumstück in der Plica lata, und zwar aus der Nähe des inneren Leistenringes. Das Ligamentumstück wurde in natürlicher Länge auf einem Holzbrett befestigt und in 10\% igem Formalin fixiert. Nach der Einbettung in Celloidin verfertigte man Quer- und Längsschnitte von $15 \mu$ Dicke und färbte sie mit Hämatoxylin und Eosin, mit dem WEIGERTschen Resorcinfuchsin, sowie zur Darstellung der Retikulumfasern nach von SANO modifizierten BIELSCHOWSKYschen Methode.

Der Flächeninhalt des Querschnittes des Ligamentes wurde mit dem Planimeter an dem auf Papier in 20facher Vergrößerung gezeichneten Bild ermittelt. Man nahm den Durchschnitt der Werte von jedem Lebensalter.

Man bezeichnete den inneren Teil des rundlichen Querschnittes des Ligamentums mit zwei Drittel des Halbmessers des ganzen Ligamentes als den Centralteil des Ligamentes und den übrigen Teil als seinen Randteil. Es wurde die Dicke der 10 dicksten Kollagen- und 10 dicksten glatten Muskelfasern an 10 Stellen im Central- und Randteil des Querschnittes gemessen und der Durchschnitt aus 100 Bestimmungen genommen. Dabei wurde der Flächeninhalt des unregelmäßig gestalteten Querschnittes der Kollagenfasern an den $1500 \mathrm{fach}$ vergrößerten Bildern 
mit dem Planimeter bestimmt. Die fast rundliche Querschnittsfläche der glatten Muskelfasern ließ sich einfach mit dem Okularmikrometer messen, und aus dem Wert des Halbmessers wurde der Flächeninhalt berechnet. Man bestimmte ferner die ganze Zahl der unter dem Lichtmikroskop sichtbaren Kollagenfasern, elastischen Fasern und glatten Muskelfasern in der Flächeneinheit $(0.1 \mathrm{~mm})^{2}$ des Central- und Randteil des Bandes.

Man maß weiterhin die ungefähre Menge der Kollagenfasern und glatten Muskelfasern in $(0.1 \mathrm{~mm})^{2}$ des Central- und Randteiles des Querschittes des Ligamentes, wie folgt. Wenn man den Mittelwert der Dicke der dicksten Kollagenfasern bzw. der glatten Muskelfasern in der Flächeneinheit als d und ihre Zahl als z bezeichnet, so ist die ungefähre Menge der Kollagenfasern bzw. der glatten Muskelfasern in der Flächeneinheit $\mathrm{m}=\mathrm{d} \times \mathrm{z}$.

\section{Ergebnisse.}

Die Ergebnisse der Untersuchung sind übersichtlich in den Tabellen zusammengestellt. Im folgenden seien die Befunde in allen Alterstufen ausführlicher wiedergegeben.

\section{a) Bei den Fröten.}

Bei den Föten ist das Ligamentum noch sehr dünn.

Die Kollagenfasern sind im ganzen Querschnitt des Bandes noch dünn und gering in Zahl (Abb. 7). Sie nehmen in der zweiten Hälfte der Gravidität an Dicke zu (Tabelle 1). Ihre Zahl in der Flächeneinheit des Querschnittes vermehren sich in der fetalen Zeit nicht viel, wohl aber im ganzen Querschnitt (Tabelle 2). Die Kollagenfasern finden sich im Randteil des Ligamentums in Zahl immer 13-16fach mehr als im Centralteil.

Die elastischen Fasern, die sich zwischen den Kollagenfasern, zwischen den

Tabelle 1. Die Dicke $\left(\mu^{2}\right)$ und Zahl der dicksten Kollagenfasern, der Diameter ( $\boldsymbol{k})$ der dicksten glatten Muskelfasern sowie die Zahl der Kollagenfasern, elasti schen Fasern und glatten Muskelfasern in $(0.1 \mathrm{~mm})^{2}$ im Ligamentum teres uteri.

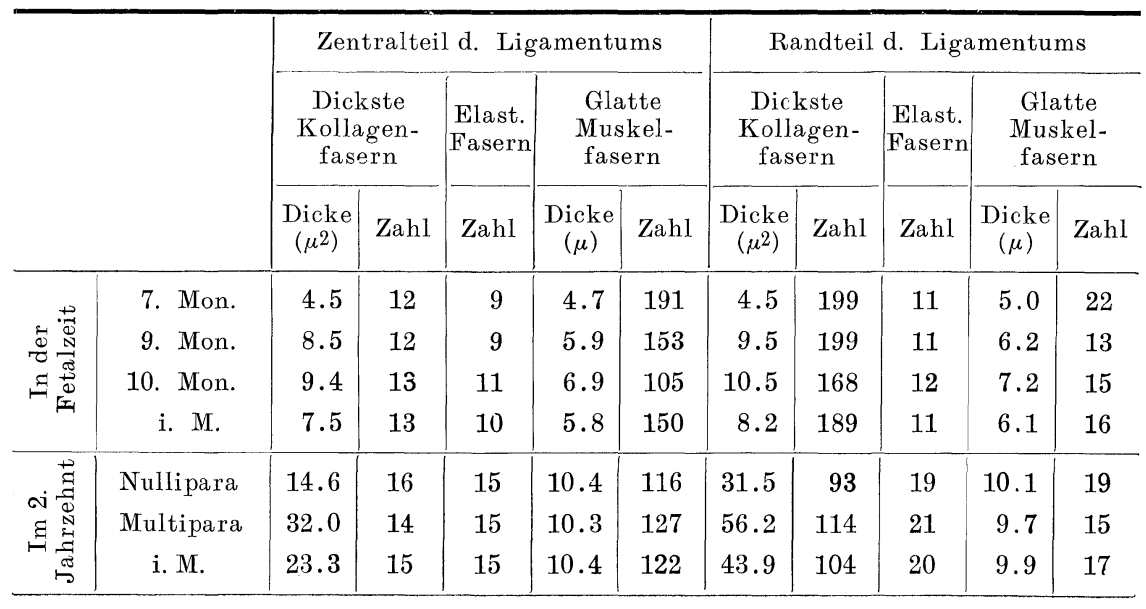


Baù des Ligamentum teres uteri in verschiedenem Lebensalter.

\begin{tabular}{|c|c|c|c|c|c|c|c|c|c|c|c|}
\hline \multirow{3}{*}{ 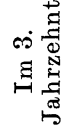 } & Nullipara & 43.5 & 14 & 18 & 11.2 & 132 & 69.0 & 95 & 24 & 11.3 & 14 \\
\hline & Multipara & 39.3 & 14 & 17 & 10.2 & 137 & 60.9 & 123 & 22 & 10.3 & 16 \\
\hline & i. M. & 41.4 & 14 & 17 & 10.7 & 134 & 65.0 & 109 & 23 & 10.8 & 15 \\
\hline \multirow{3}{*}{ 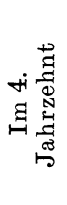 } & $\begin{array}{l}\text { 1-3 mal } \\
\text { Gebärende }\end{array}$ & 29.6 & 20 & 19 & 10.8 & 143 & 29.4 & 108 & 24 & 10.8 & 19 \\
\hline & $\begin{array}{c}\text { mehr als } \\
3 \text { mal } \\
\text { Gebärende }\end{array}$ & 29.6 & 13 & 20 & 9.3 & 173 & 29.2 & 137 & 27 & 9.2 & 18 \\
\hline & i. M. & 29.6 & 17 & 20 & 10.1 & 158 & 29.3 & 123 & 26 & 10.0 & 19 \\
\hline \multirow{4}{*}{ 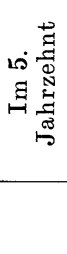 } & $\begin{array}{c}4 \text { mal } \\
\text { Gebärende }\end{array}$ & 15.5 & 16 & 37 & 8.7 & 230 & 22.9 & 161 & 45 & 8.7 & 22 \\
\hline & $\begin{array}{c}6 \text { mal } \\
\text { Gebärende }\end{array}$ & 10.9 & 15 & 34 & 9.1 & 268 & 6.4 & 208 & 41 & 9.8 & 23 \\
\hline & i. $M$. & 13.2 & 16 & 35 & 8.9 & 249 & 14.7 & 184 & 43 & 9.3 & 23 \\
\hline & $\begin{array}{c}\text { Im höheren } \\
\text { Alter }\end{array}$ & 10.4 & 15 & 138 & 6.6 & 199 & 12.4 & 182 & 156 & 6.7 & 21 \\
\hline
\end{tabular}

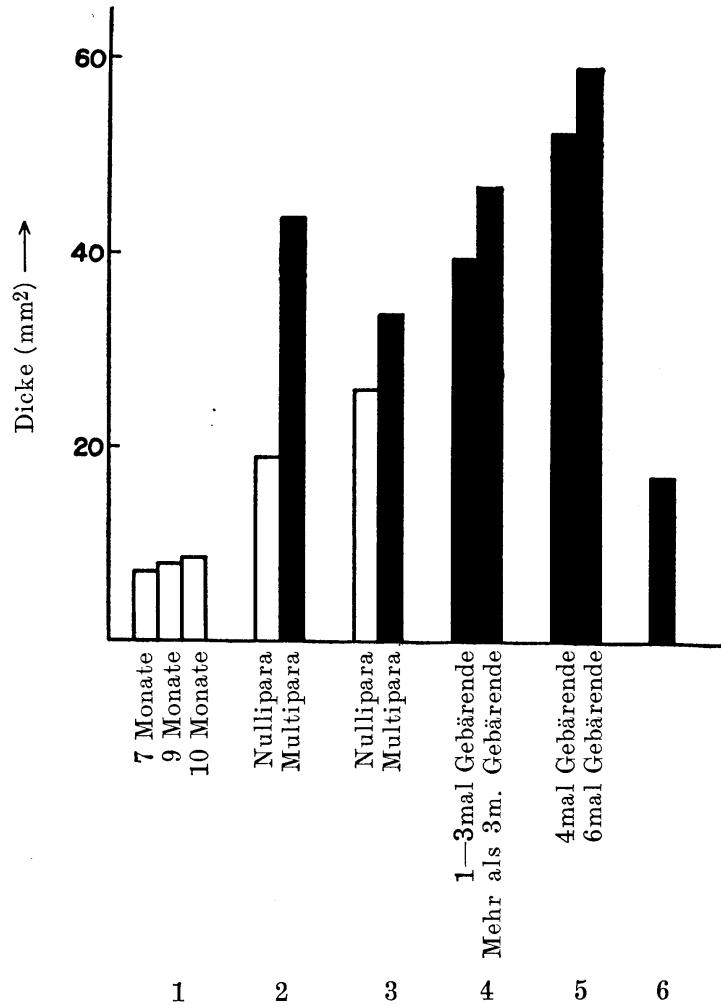

Abb. 1. Der Flächeninhalt des Querschnittes des Ligamentums $\left(\mathrm{mm}^{2}\right)$. 1. in der Fetalzeit, 2. im 2. Jahrzehnt, 3. im 3. Jahrzehnt, 4. im 4. Jahrzehnt, 5. im 5. Jahrzehnt, 6. im höheren Alter. 
Tabelle 2. Die Dicke des Ligamentum teres uteri, sowie die ungefähre $(0.1 \mathrm{~mm})^{2}$, im Central- und Randteil und im

\begin{tabular}{|c|c|c|c|c|c|c|}
\hline & & \multirow{3}{*}{$\begin{array}{l}\text { Dicke d. } \\
\text { Lig. } \\
\left(\mathrm{mm}^{2}\right)\end{array}$} & \multicolumn{4}{|c|}{ Kollagenfasern } \\
\hline & & & \multicolumn{2}{|c|}{$\begin{array}{l}\text { Ungëfahre Menge in } \\
\text { d. Flächeneinheit } \\
\qquad\left(\mu^{2}\right)\end{array}$} & \multirow{2}{*}{$\begin{array}{c}\text { Ungefähre } \\
\text { Menge im } \\
\text { Centralteil } \\
\left(\mu^{2}\right)\end{array}$} & \multirow{2}{*}{$\begin{array}{c}\text { Ungefähre } \\
\text { Menge im } \\
\text { Randteil } \\
\quad\left(\mu^{2}\right)\end{array}$} \\
\hline & & & $\underset{\text { im }}{\text { Centralteil }}$ & $\stackrel{\text { im }}{\text { Randteil. }}$ & & \\
\hline \multirow{4}{*}{ 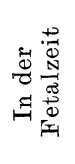 } & 7. Mon. & 7.2 & 56 & 897 & 17900 & 358800 \\
\hline & 9. Mon. & 7.8 & 105 & 1886 & 36900 & 810900 \\
\hline & 10. Mon. & 8.5 & 119 & 1760 & 45400 & 827100 \\
\hline & i. $\mathrm{M}$. & 7.8 & 94 & 1546 & 32800 & 664700 \\
\hline \multirow{3}{*}{ 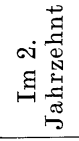 } & Nullipara & 19.0 & 241 & 2920 & 192700 & 3212100 \\
\hline & Multipara & 43.3 & 445 & 6429 & 854000 & 15494600 \\
\hline & i. M. & 33.6 & 354 & 4548 & 527800 & 8504800 \\
\hline \multirow{3}{*}{  } & Nullipara & 26.0 & 587 & 6534 & 675400 & 9474700 \\
\hline & Multipara & 33.7 & 562 & 7503 & 843000 & 14030400 \\
\hline & i. M. & 30.6 & 576 & 7085 & 782700 & 12044500 \\
\hline \multirow{3}{*}{ 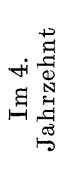 } & $\begin{array}{c}1-3 \text { mal } \\
\text { Gebährende }\end{array}$ & 39.5 & 598 & 3187 & 1046300 & 7011400 \\
\hline & $\begin{array}{c}\text { mehr als } 3 \text { mal } \\
\text { Gebährende }\end{array}$ & 47.0 & 391 & 4006 & 816600 & 10456200 \\
\hline & i. $\mathrm{M}$. & 44.0 & 494 & 3597 & 963900 & 8812700 \\
\hline \multirow{4}{*}{ ن } & $\begin{array}{l}4 \text { mal } \\
\text { Gebährende }\end{array}$ & 52.3 & 254 & 3682 & 602300 & 10678700 \\
\hline & $\begin{array}{c}6 \text { mal } \\
\text { Gebährende }\end{array}$ & 59.0 & 162 & 1331 & 425500 & 4366300 \\
\hline & i. M. & 55.0 & 205 & 2711 & 499200 & 8294700 \\
\hline & $\begin{array}{l}\text { Im höheren } \\
\text { Alter }\end{array}$ & 17.0 & 151 & 2262 & 113100 & 2148700 \\
\hline
\end{tabular}

Muskelbündeln und um die Gefäße finden, sind in der Fetalzeit sehr dünn und durchschnittlich gleich dick wie die Kollagenfasern. Sie laufen selbst im lockeren Gewebe fast nicht geschlängelt (Abb. 8 und 9). Mit dem Fortschreiten der Monate vermehren sich die elastischen Fasern in der Flächeneinheit des Querschnittes um weniges. Ihre Zahl in der Flächeneinheit ist immer etwas mehr im Randteil als im Centralteil.

Die Retikulumfasern kommen zwischen den glatten Muskelfasern reichlich vor. Ihre Zahl in der Flächeneinheit nimmt in der Fetalzeit zu, ihre Dicke aber kaum.

Die glatten Muskelfasern machen den größeren Teil der Elemente des Ligamentes aus (Abb. 7). Ihre Dicke nimmt in der Fetalzeit immer mehr zu (Tabelle 1).

b) Im 2. Jahrzehnt.

Das Ligamentum im 2. Lebensdezennium ist, wie in Tabelle 2 gezeigt ist, bei 
Menge der Kollagenfasern und glatten Muskelfasern in der Flächeneinheit ganzen Querschnitt des Ligamentums.

\begin{tabular}{|c|c|c|c|c|c|}
\hline \multirow{3}{*}{$\begin{array}{l}\text { Ungefähre } \\
\text { Menge im } \\
\text { ganzen } \\
\text { Querschnitt } \\
\quad(\mu 2)\end{array}$} & \multicolumn{5}{|c|}{ Glette Muskelfasern } \\
\hline & \multicolumn{2}{|c|}{$\begin{array}{l}\text { Ungef ähre Menge in d. } \\
\text { Flächeneinheit }\left(\mu^{2}\right)\end{array}$} & \multirow{2}{*}{$\begin{array}{c}\text { Ungefähre } \\
\text { Menge im } \\
\text { Centralteil } \\
\left(\mu^{2}\right)\end{array}$} & \multirow{2}{*}{$\begin{array}{c}\text { Ungefähre } \\
\text { Menge im } \\
\text { Randteil } \\
\quad\left(\mu^{2}\right)\end{array}$} & \multirow{2}{*}{$\begin{array}{l}\text { Ungefähre } \\
\text { Menge im } \\
\text { ganzen } \\
\text { Querschnitt } \\
\quad\left(\mu^{2}\right)\end{array}$} \\
\hline & $\stackrel{\text { im }}{\text { Centralteil }}$ & $\begin{array}{l}\text { im } \\
\text { Randteil }\end{array}$ & & & \\
\hline 376600 & 896 & 108 & 286700 & 43000 & 329700 \\
\hline 847800 & 905 & 78 & 316600 & 33300 & 349900 \\
\hline 872500 & 724 & 106 & 275300 & 49700 & 325000 \\
\hline 697500 & 868 & 99 & 303900 & 42500 & 346400 \\
\hline 3404800 & 1211 & 188 & 969500 & 206700 & 1175200 \\
\hline 16348600 & 1309 & 147 & 2513500 & 353100 & 2866500 \\
\hline 9032500 & 1267 & 167 & 1887400 & 312900 & 2200200 \\
\hline 10150100 & 1478 & 157 & 1700200 & 227800 & 1928000 \\
\hline 14873400 & 1367 & 161 & 2050500 & 300500 & 2351000 \\
\hline 12827200 & 1438 & 160 & 1955800 & 277700 & 2227500 \\
\hline 8057700 & 1544 & 208 & 2702700 & 458500 & 3161200 \\
\hline 11272700 & 1606 & 169 & 3362600 & 441900 & 3804500 \\
\hline 9776500 & 1596 & 189 & 3111800 & 463100 & 3574900 \\
\hline 11281000 & 1999 & 191 & 4658400 & 555100 & 5213400 \\
\hline 4791800 & 2439 & 228 & 6389700 & 748800 & 7138500 \\
\hline 8794000 & 2215 & 211 & 5405100 & 646300 & 6051400 \\
\hline 2261800 & 1316 & 138 & 987000 & 131100 & 1118100 \\
\hline
\end{tabular}

den Nulliparen etwa 2.4fach und bei den Multiparen etwa 5 fach dicker als in der Fetalzeit.

Die Dicke der dicksten Kollagenfasern von Nulliparen im 2. Lebensdezennium sind, wie in Tabelle 1 zu sehen ist, 2.4 fach größer als in der Fetalzeit. Die Fasern sind bei der Multipara dicker und in weitaus größerer Menge vorhanden als bei der Nullipara (Abb. 12). Sie sind bei der Nullipara im Centralteil des Ligamentes mehr vorhanden als im Randteil, bei der Multipara aber umgekehrt.

In Übereinstimmung mit den Befunden von MIYAZAKI (1931) sind die elastischen Fasern des Ligamentums bei der Nullipara im 2. Lebensdezennium etwa 2 mal dicker als bei der Fetalzeit (Abb. 13, 14 und 15). Sie schlängeln sich auch stärker und sind mit Resorcinfuchsin stärker färbbar. Bei der Núllipara und auch bei der Multipara sind die elastischen Fasern im Randteil dicker, Zahlreicher in 
der Flächeneinheit des Querschnittes und stärker geschlängelt als im Centralteil. Bei der Multipara entwickeln sich die elastischen Fasern noch stärker und sind mit Resorcinfuchsin noch stärker färbbar (vgl. Abb. 13 und 15).

Retikulumfasern sind zwischen den glatten Muskelfasern zu sehen, aber weniger als bei den Föten.

Die glatten Muskelfasern sind bei der Nullipara im 2. Jahrzehnt etwa 2 fach dicker als bei der Fetalzeit. Sie sind bei der Multipara dünner als bei der Nullipara. Die Zahl der glatten Muskelfaesrn in der Flächeneinheit des Querschnittes des Ligamentes ist bei der Nullipara im Randteil und bei der Multipara im Centralteil größer. Die Menge der glatten Muskelfasern im ganzen Querschnitt des Ligamentums beträgt ferner bei der Multipara mehr als das Doppelte als bei Nullipara (Tabelle 2).

c) Im 3. Jahrzehnt.

Wie in Tabelle 1. angegeben ist, beträgt die Dicke der dicksten Kollagenfasern im Centralteil des Ligamentums durchschnittlich 4.1.4 $\mu^{2}$ und im Randteil $64.9 \mu^{2}$ während des 3. Lebensdezenniums. Die Kollagenfasern sind somit in dieser Zeit des r,ebens am dicksten (Tabelle 1 und Abb. 2). Ihre Menge im ganzen Ligamentum

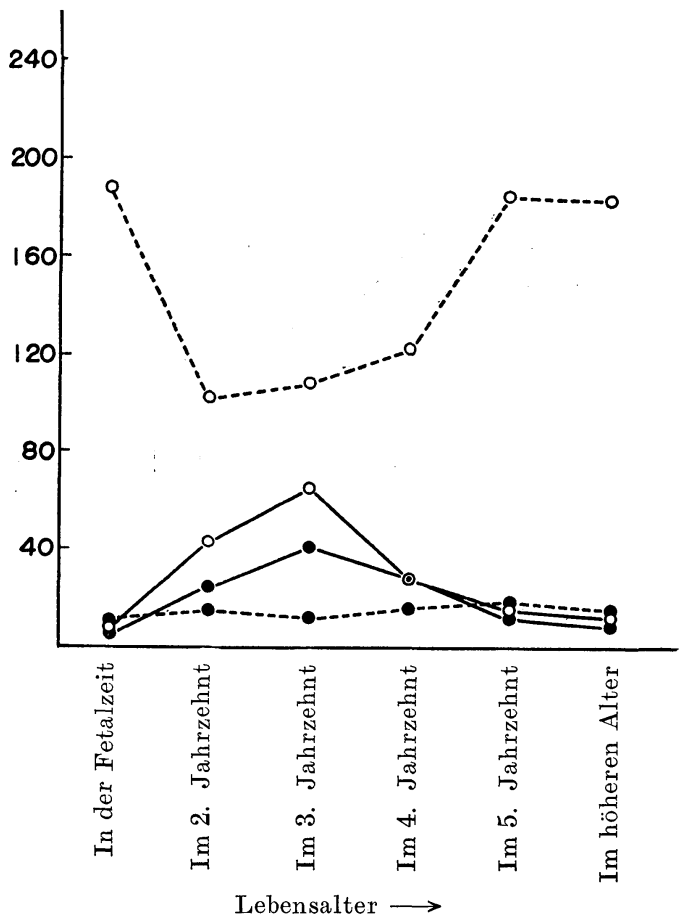

Abb. 2. Die dicke $\left(\mu^{2}\right)$ der Kollagenfasern und ihre Zahl in der Flächeneinheit des Querschnittes des Ligamentums $(0.1 \mathrm{~mm})^{2}$.

Dicke der Kollagenfasern im Centralteil, ○—— Dicke der Kollagenfasern im Randteil, --.-- Zahl der Kollagenfasern im Centralteil, ○...... Zahl der Kollagenfasern im Randteil. 
ist auch am größten.

Die elastischen Fasern entwickeln sich auch in dieser Zeit besser als vorher (Abb. 3, 19 und 20).

Die Retikulumfasern sind immer zwischen den glatten Muskelfasern zu sehen.

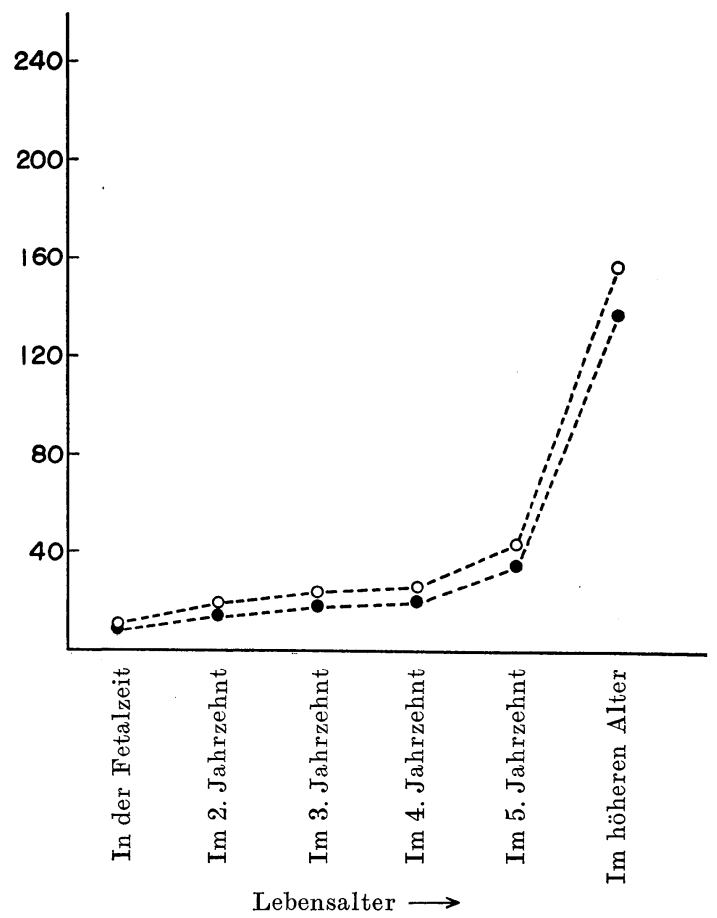

Abb. 3. Die Zahl der elastischen Fasern in der Flächeneinheit des Querschnittes des Ligamentums $(0.1 \mathrm{~mm})^{2}$. Zahl der elastichen Fasern im Centralteil, ○..... O Zahl der elastischen Fasern im Randteil.

Die glatten Muskelfasern sind auch in dieser Zeit des Lebens am dicksten (Abb. 4). Sie sind bei der Multipara etwas dünner als bei der Nullipara. Ihre Zahl in der Flächeneinheit ist im Centralteil etwa $10 \mathrm{fach}$ größer als im Randteil. Im ganzen Querschnitt des Ligamentums ist die Menge der glatten Muskelfasern bei der Nullipara im 3. Lebensdezennium etwa $1.6 \mathrm{fach}$ größer als im 2. Lebensdezennium (Tabelle 2).

d) Im 4. Jahrzehnt.

Die Dicke des Ligamentums wird nun etwa 1.4fach dicker als im vorigen Jahrzehnt (Tabelle 2). Die Dicke der dicksten Kollagenfasern reduziert sich aber im Centralteil des Ligamentums bis zu etwa $2 / 3$ und im Randteil bis zu etwa $1 / 2$. Ihre Zahl in der Flächeneinheit im Centralteil des Querschnittes ist am größten im ganzen Leben, und sie ist im Randteil größer als bisher. Mit der Wiederholung der Geburt werden sie besonders im Randteil verhältnismäßig zahlreicher. Die Menge der Kollagenfasern im Ligamentum nimmt bis etwa $3 / 4$ von derjenigen im 


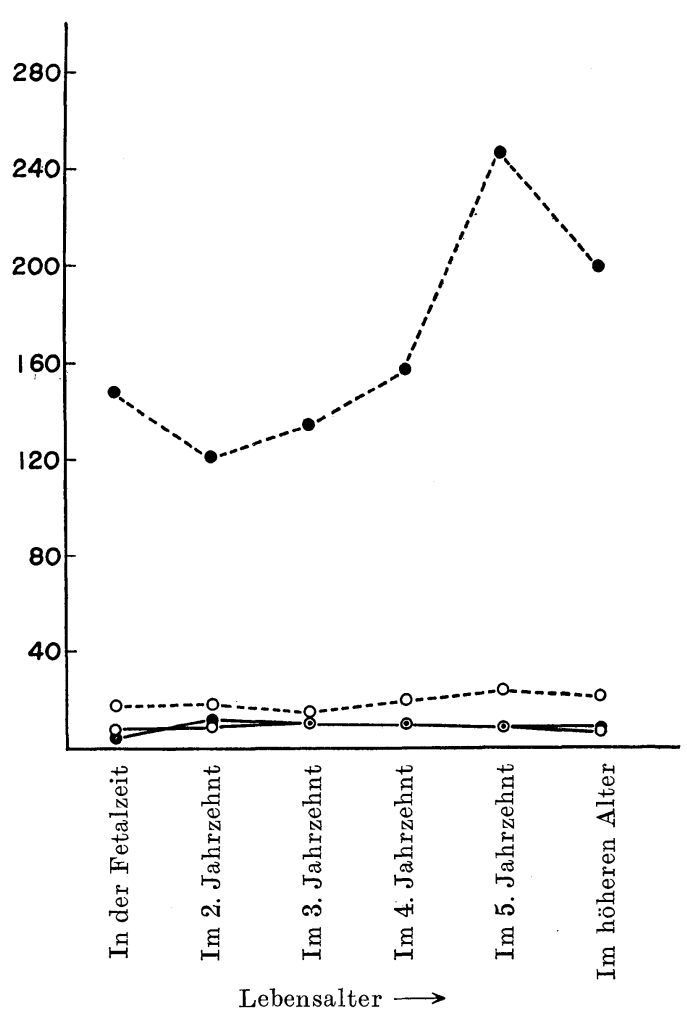

Abb. 4. Die Dicke ( $\mu$ ) der glatten Muskelfasern in der Flächeneinheit des Querschnitts des Ligamentumes $(0.1 \mathrm{~mm})^{2}$. —— Dicke der glatten Muskelfasern im Centralteil, $\bigcirc-O$ Dicke der glatten Muskelfasern im Randteil, @-.... Zahl der glatten Muskelfasern im Centralteil, ○..... Z Zahl der glatten Muskelfasern im Randteil.

3. Lebensdezennium ab. Mit der Geburt vermehrt sich aber die Menge (Tabelle 2).

Die elastischen Fasern werden dicker und in der Flächeneinheit zahlreicher und knäueln sich häufig (Abb. 24).

Die glatten Muskelfasern werden etwas dünner (s. Tabelle 1 und vgl. Abb. 18 und 23; s. ferner Abb. 4).

Die Menge der glatten Muskelfasern im ganzen Querschnitt des Ligamentums wird etwa 1.6fach größer als im vorigen Lebensdezennium (s. Tabelle 2). Mit der Geburt nimmt sie zu.

e) Im 5. Jahrzehnt.

Das Ligamentum wird nun am dicksten (Tablle 2 und Abb. 1).

Die Dicke der dicksten Kollagenfasern verringert sich durchschnittlich auf etwa $1 / 2$ von derjenigen im 4. Lebensdezenniums (Abb. 2). Sie wird auch mit der Wiederholung der Geburt durchschnittlich kleiner. Ihre Zahl in der Flächeneinheit nimmt mit der Geburtswiederholung im Centralteil ab, aber im Randteil zu. Die Menge der Kollagenfasern wird etwas geringer als im vorigen Lebensdezennium 


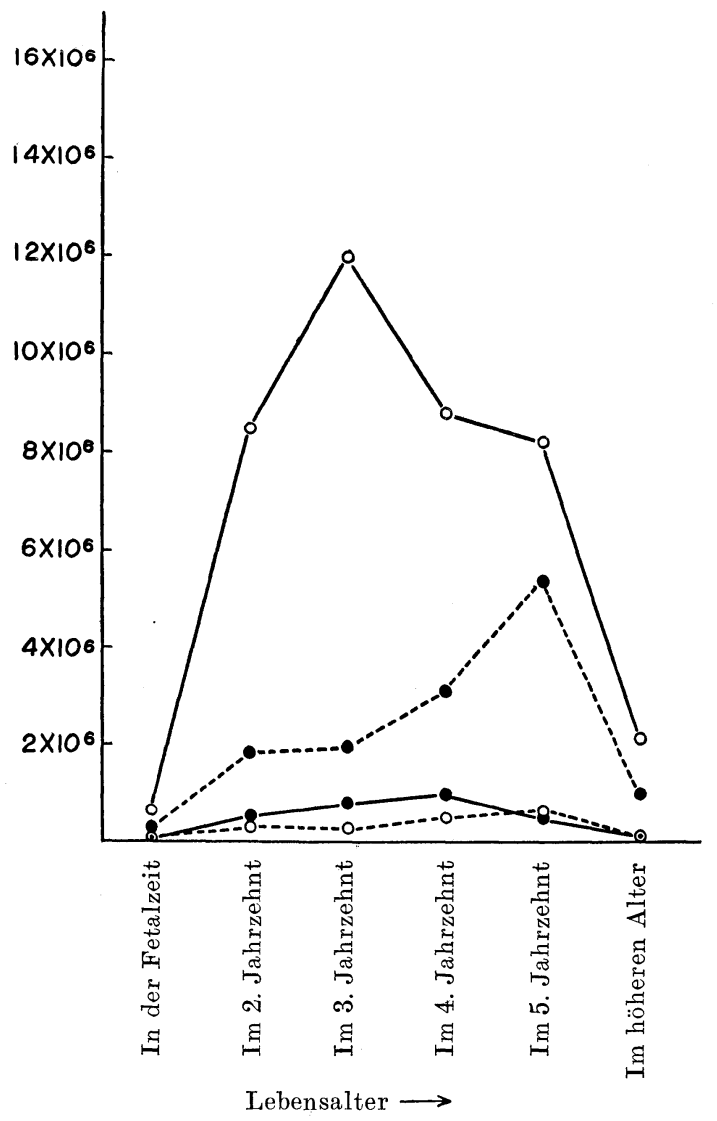

Abb. 5. Die Menge der Kollagenfasern und glatten Muskelfasern im Centralund Randteil des Querschnittes des Ligamentums. - $\longrightarrow$ Menge der Kollagenfasern im Centralteil, $\mathrm{O}-\mathrm{O}$ Menge der Kollagenfasern im Randteil, -..... Menge der glatten Muskelfasern im Centralteil, O...... Menge der glatten Muskelfasern im Randteil.

(Tabelle 2).

Die elastischen Fasern in der Flächeneinheit sind etwa $1.5 \mathrm{fach}$ zahlreicher als im 4. Lebensdezennium (Abb. 3, 26 und 27)

Die Dicke der glatten Muskelfasern ist durchschnittlich dünner als vorher (Abb. 4); ihre Zahl in der Flächeneinheit ist sowohl im Centralteil als auch im Randtєil des Ligamentums am größten unter allen Lebensaltern. Ihre Zahl vermehrt sich mit der Geburt. Die Menge der glatten Muskelfasern im ganzen Querschnitt des Ligamentums ist am größten im ganzen Lauf des Lebens. Sie nimmt aber auch mit der Geburt zu (Tabelle 2).

f) Im höheren Alter

Das Ligamentum verdünnt sich beträchtlich, und sein Querschnitt beträgt $17 \mathrm{~mm}^{2}$ im Mittel. 


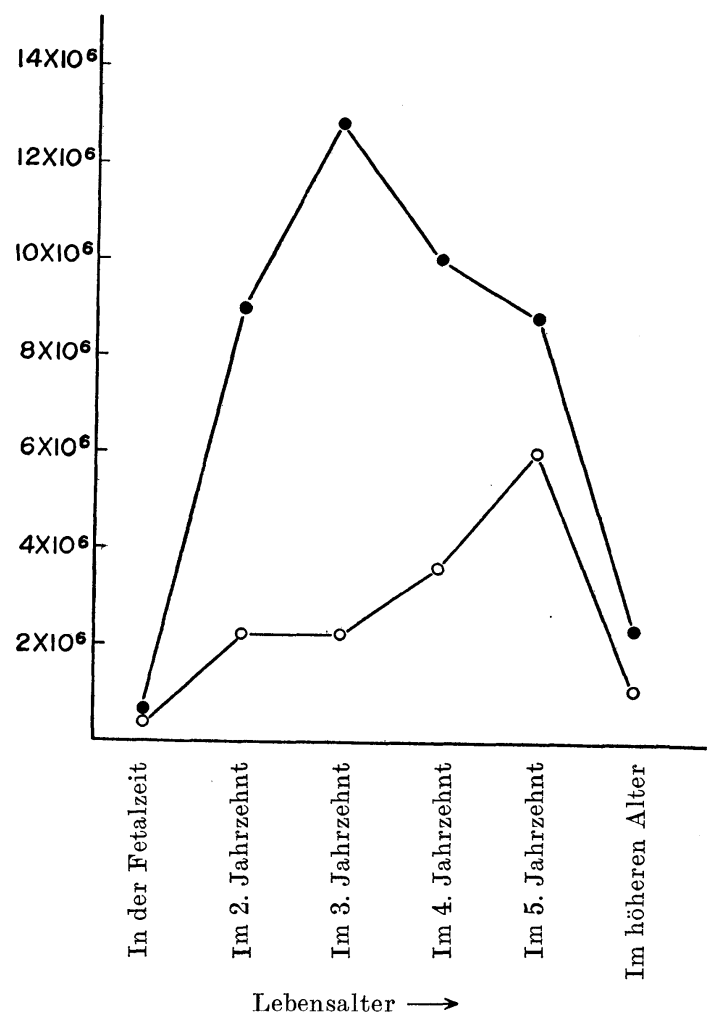

Abb. 6. Die Menge der Kollagenfasern und glatten Muskelfasern im ganzen Querschnittes des Ligamentums. Menge der glatten Muskelfasern.

Die dicksten Kollagenfasern betragen im Centralteil $10.4 \mu^{2}$ und im Randteil $12.4 \mu^{2}$ (Abb. 2, 28 und 29). Ihre Zahl in der Fläsheneinheit ist aber wie im vorigen Lebensdezennium. Die Menge der Kollagenfasern im ganzen Querschnitt des Ligmentums ist am geringsten nach der Geburt (Tabelle 2).

Die elastischen Fasern laufen nicht mehr viel geschlängelt. Ihre Zahl bleibt fast wie vorher. Die Färbbarkeit der Fasern mit Resorcinfuchsin erweist sich als besser als bei jüngeren Frauen.

Die Zahl der Retikulumfasern zwischen den glatten Muskelfasern ist nunmehr sehr gering.

Die glatten Muskelfasern sind durchschnittlich am dünnsten seit der Geburt. Sie finden sich weniger zahlreich als im letzten Lebensdezennium (Abb. 4). Ihre Menge im ganzen Querschnitt des Ligamentums ist, wie man es in Tabelle 2 sieht, am kleinsten nach der Geburt (s. ferner Abb. 6).

\section{Auswertung}

a) Mit dem fetalen und postnatalen Altern sowie mit der Geburt wird das 
Ligamentum teres uteri dicker und erreicht im 5. Lebensdezennium den Höhepunkt, um dann wieder dünner und endlich am dünnsten im ganzen postnatalen Leben zu werden.

b) Die Kollagenfasern.

Man sieht im Ligamentum mit dem Fortschreiten der fetalen und postnatalen Zeit immer dickere Kollagenfasern. Ihre Dicke erreicht aber das Maximum im 3. Lebensdezennium, darauf verringert sie sich allmählich. Ihre Zahl in der Flächeneinheit des Querschnittes nimmt nach der Geburt mit den Lebensjahren zu, um im 5. Lebensdezennium am größten zu werden. Bei den Föten kommen die Kollagenfasern im Randteil und im 4. Lebensdezennium im Centralteil reichlicher vor. Die Kollagenfasern werden mit Wiederholnng der Geburt immer dünner.

Die Menge der Kollagenfasern im ganzen Ligamentum vermehren sich mit der Zeit und erreichen ihr Maximum im 3. Lebensdezeunium, um dann wieder abzunehmen.

c) Die elastischen Fasern.

Die Dicke, die Zahl in der Flächeneinheit, die Schlängelung im Gewebe und die Färbbarkeit mit Resorcinfuchsin der elastischen Fasern vermehren und verstärken sich fortwährend in der fetalen und postnatalen Zeit und auch bei der Geburt. Sie entwickeln sich im Randteil des Ligamentums besser als im Centralteil. Am zahlreichsten kommen sie im hohen Alter und zeigen die stärkste Färbbarkeit mit Resorcinfuchsin, aber schlängeln sich im Gewebe weniger.

d) Die Retikulumfasern.

Die Retikulumfasern zwischen den glatten Muskelfasern des Ligamentes verringern sich im hohen Alter.

e) Die glatten Muskelfasern.

Die glatten Muskelfasern werden im Laufe des fetalen und postnatalen Lebens immer dicker, bis sie vom 3 . Lebensdezennium ab wieder dünner werden. Sie sind bei der Multipara durchschnittlich um ein Geringes dünner als bei der Nullipara von demselben Lebensdezennium, nehmen aber an Zahl etwas zu. Die ganze Menge der glatten Muskelfasern vermehren sich immer bis zum 5. Lebensdezennium und reduzieren sich dann im höheren Alter.

\section{Zusammenfassung.}

Das Ligamentum teres uteri wurde in der Plica lata uteri kurz vor dem Eintreten in den Leistenkanal untersucht.

1. Das Ligamentum verdickt sich bis zum 5. Lebensdezennium und wird dann wieder dünner.

2. Die Dicke der dicksten kollagenfasern im Ligamentum nimmt bis zum 3. Lebensdezennium zu. Sie werden darauf dünner. Bei der Geburt verringert sich ihre Dicke ein wenig. Die ganze Menge der Kollagenfasern im Ligamentum nimmt mit dem Altern und mit der Wiederholung der Geburt gewöhnlich zu.

3. Die elastischen Fasern entwickeln sich mit dem Fortschreiten der Zeit und der Wiederholung der Geburt in Bezug auf die Dicke, Zahl, Schlängelung sowie auf die Färbbarkeit mit Resorcinfuchsin. 
4. Die Retikulumfasern finden sich am meisten zwischen den glatten Muskelfasern. Sie nehmen mit der Zeit ab.

5. Die Dicke der glatten Muskelfasern im Ligamentum vermehren sich mit der fetalen und postnatalen Zeit bis zum 3. Lebensdezennium und reduzieren sich dann allmählich. Sie sind bei den Multiparen etwas dünner. Die ganze Menge der glatten Muskelfasern im Ligament nimmt mit der Zeit und durch die Geburt zu, bis sie sich vom 5 . Lebenensdezennium ab wieder verringert.

\section{内 容自抄。}

子宮広間膜内で鼠径管に大る少し前の子宮円甞の部は 50 才代で最む太くなり， 以後再び細くなる．30才代までは索内に膠原線維のますます太いものが現われる が，それ以後は太いものも細くなる。よく見ると，分㚾のときには膠原線維が細 くなるが，索内の全量は年令と分㚾とともに増す。また年令と分婏とともに弾性 線維の太さ, 数, 迁曲の度とレゾルチンフクシンでの染色性が増す。平滑筋線維 の間にある細網線維は年令と共に減ずる.

索内の平滑筋線維の太さは30才代までは増し, 以後漸減する。経産者ではやや 細い. 索内の平滑筋線維の全量は年令と分㚾とともに増し，50才代で最高に達し， それから漸減する。

\section{Literatur.}

Keiffer, H. : Études sur l'histophysiologie uterine. Recherches sur le ligament rond. Gynec. et Obstetr. 11 (1925). - Koelliker, A.: Handbuch der Gewebelehre des Menschen. Leipzig, 1902. - Miyazaki, N.: Histologische Untersuchung des Lig. rotundum uteri. I. Über die elastischen Fasern im Lig. rotundum. (Jap.) Nippon Fujinka Gk. Z. 26 (1931). Sano, Y.: Versuch, die Silberimprägnationsmethode von Gitterfasern zu verbessern. Arch. hist. jap. 3 (1952). 
Tafelabbildungen. 


\section{Erläuterung zu den Tafelabbildungen.}

Abb. 7. Übersicht über den Querschnitt des Ligamentums $(22 \times)$ und der Längsschnitt des Centralteiles desselben $(110 \times)$. Aus einem Fötus von 7 Monaten. Färbung mit Hämatoxylin und Eosin.

Abb. 8. Längsschnitt des Übergangsstelle des Cental- und Randteiles des Ligamentums. Aus einem Fötus von 7 Monaten. Färbung mit Resorcinfuchsin. $110 \times$

Abb. 9. Übersicht über den Querschnitt $(22 \times)$ und der Längsschnitt $(110 \times)$ der Übergangsstelle des Central- und Randteiles des Ligamentums. Aus einem Fötus von 10 Monaten. Färbung mit Resorcinfuchsin.

Abb. 10. Übersicht über den Querschnitt des Ligamentums. Aus einer einmal Gebärenden von 21 Jahren. Färbung mit Hämatoxylin und Eosin. $22 \times$

Abb. 11. Querschnitt des Centralteiles des Ligamentums. Aus einer Nullipara von 26 Jahren. Färbung mit Hämatoxylin und Eosin. $110 \times$

Abb. 12. Querschnitt des Centralteiles des Ligamentums. Aus einer 2mal Gebärenden von 26 Jahren. Färbung mit Hämatoxylin und Eosin. $110 \times$ 
Y. TAKEBAYASHI (1)
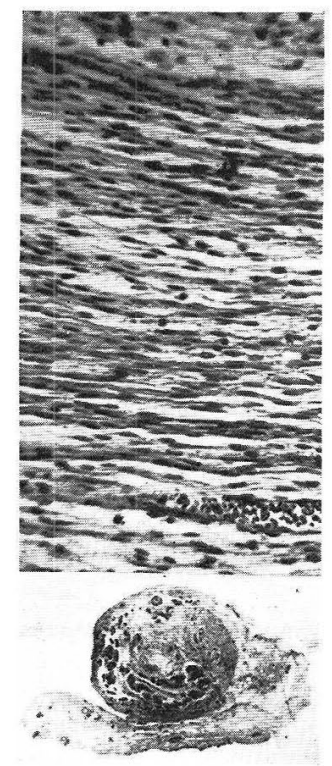

7

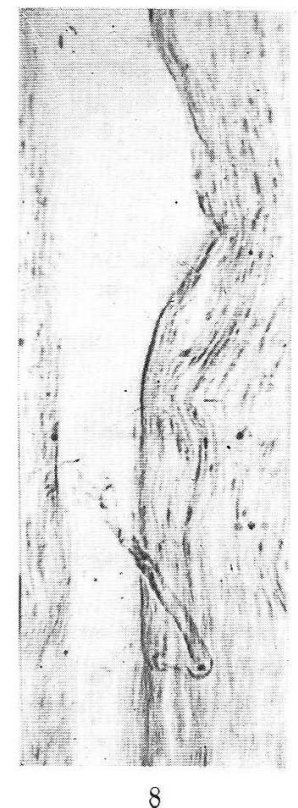

8

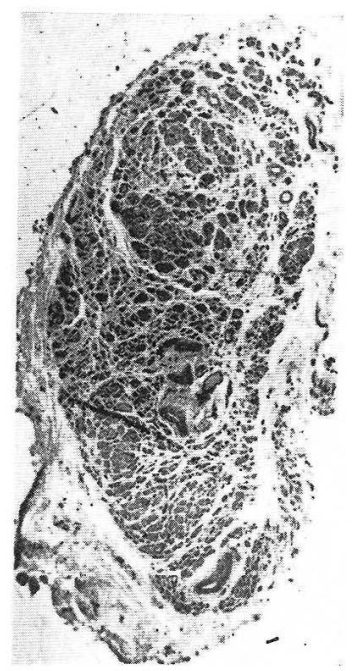

11

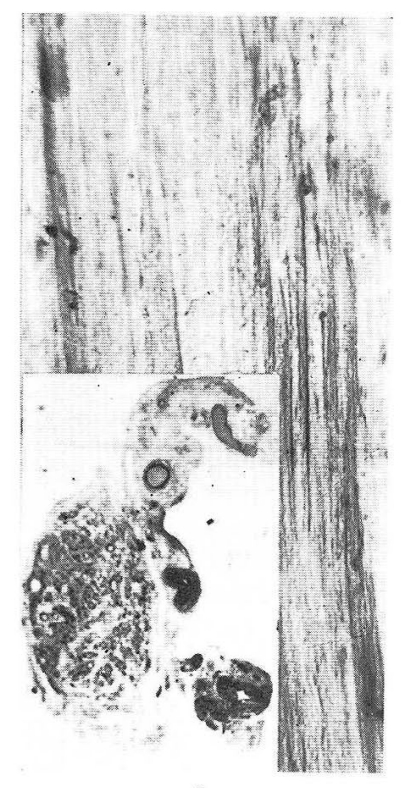

9

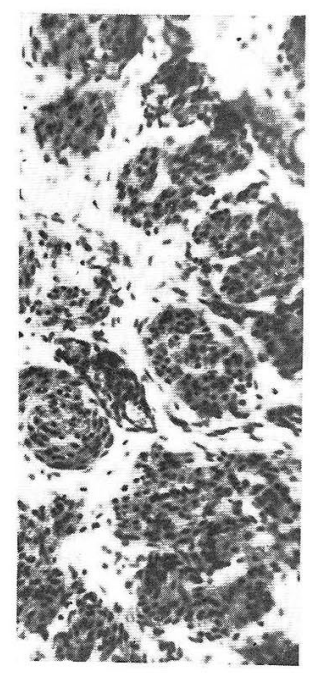

12 
Abb. 13. Längsschnitt des Randteiles des Ligamentums. Aus einer Nullipara von 22 Jahren. Färbung mit Resorcinfuchsin. $110 \times$

Abb. 14. Übersicht über den Querschnitt des Ligamentums. Aus einer einmal Gebärenden von 26 Jahren. Färbung mit Resorcinfuchsin. $22 \times$

Abb. 15. Längsschnitt des Randteiles des Ligamentums. Aus einer 2mal Gebärenden von 25 Jahren. Färbung mit Resorcinfuchsin. $110 \times$

Abb. 16. Übersicht über den Querschnitt des Ligamentums. Aus einer Nullipara von 32 Jahren. Färbung mit Hämatoxylin und Eosin. $22 \times$

Abb. 17. Übersicht über den Querschnitt des Ligamentums. Aus einer einmal Gebärenden von 36 Jahren. Färbung mit Hämatoxylin und Eosin. $22 \times$

Abb. 18. Längsschnitt des Centralteiles des Ligamentums in Abb. 17. $110 \times$ 
Y. TAKEBAYASHI (2)

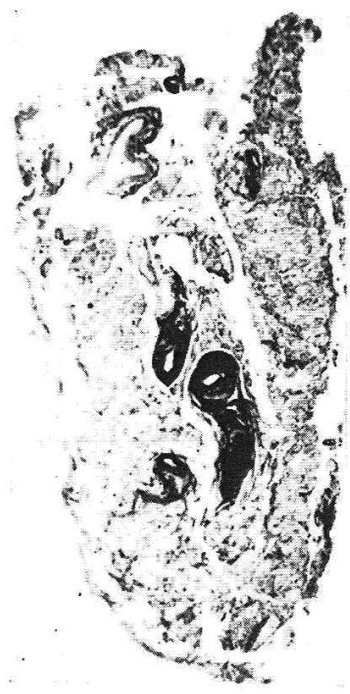

13

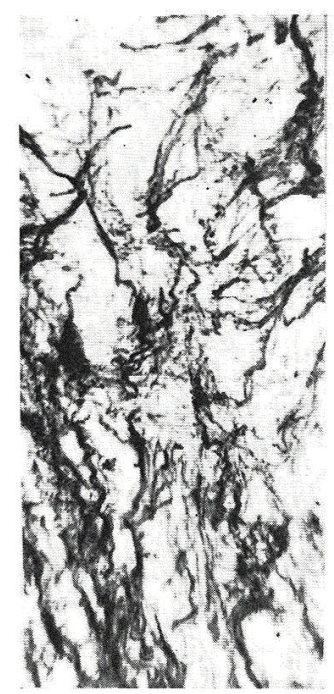

14



15

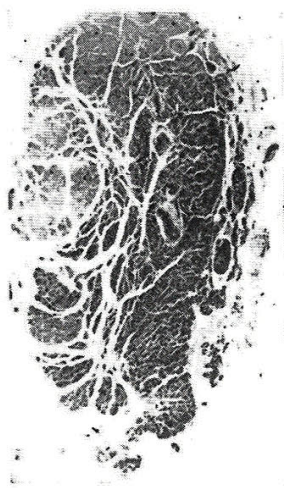

16
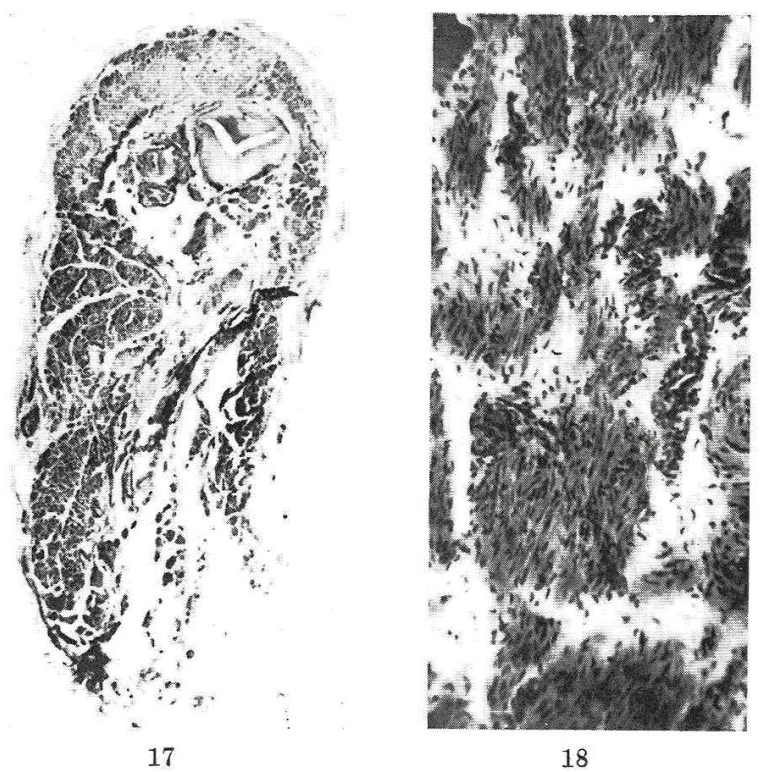

18 
Abb. 19. Übersicht über den Querschnitt des Ligamentums. Aus einer Nullipara von 33 Jahren. Färbung mit Resorcinfuchsin. $22 \times$

Abb. 20. Übersicht über den Querschnitt des Ligamentums. Aus einer 6mal Gebärenden von 34 Jahren. Färbung mit Resorcinfuchsin. $22 \times$

Abb. 21. Längsschnitt des Randteiles des Ligamentums in Abb. 20. $110 \times$

Abb. 22. Übersicht über den Querschnitt des Ligamentums. Aus einer 5 mal Gebärenden von 45 Jahren. Färbung mit Hämatoxylin und Eosin. $22 \times$

Abb. 23. Längsschnitt des Centralteiles des Ligamentums. Aus einer 3mal Gebärenden von 48 Jahren. Färbung mit Hämatoxylin und Eosin. $110 \times$

Abb. 24. Längsschnitt des Randteiles des Ligamentums. Aus einer 4 mal Gebärenden von 48 Jahren. Färbung mit Resorcinfuchsin. $110 \times$ 
Y. TAKEBAYASHI ( 3 )

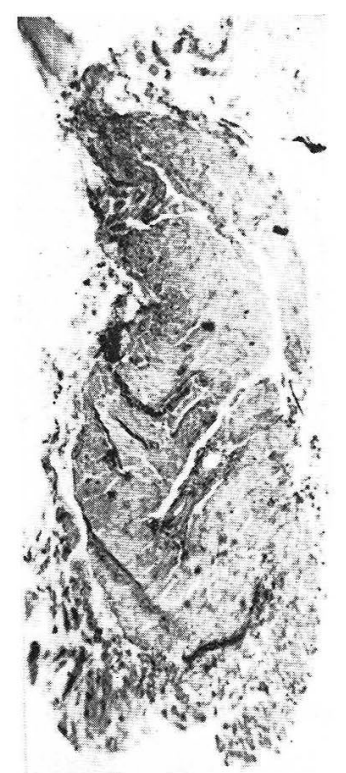

19

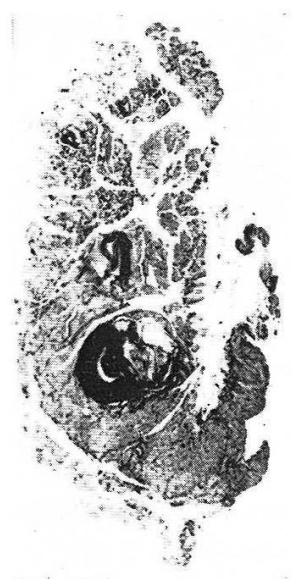

20

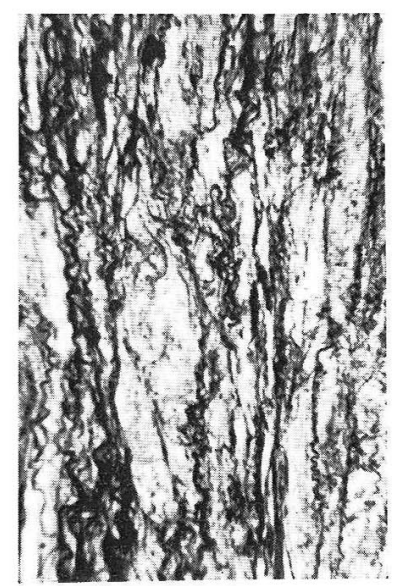

21

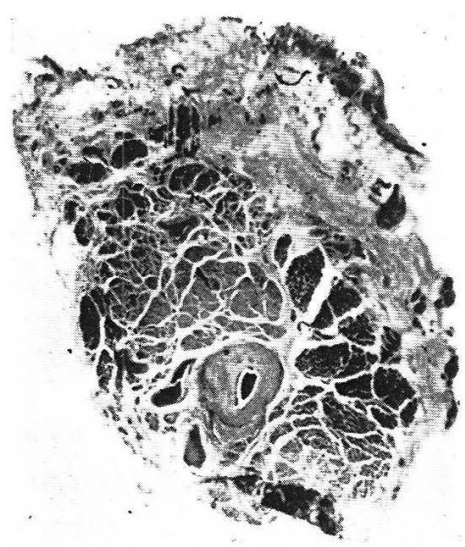

22

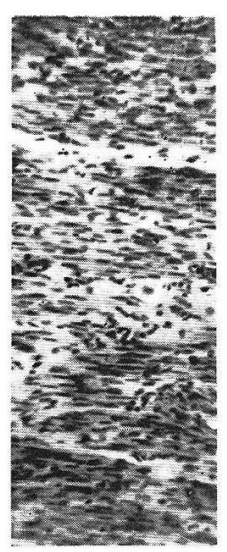

23

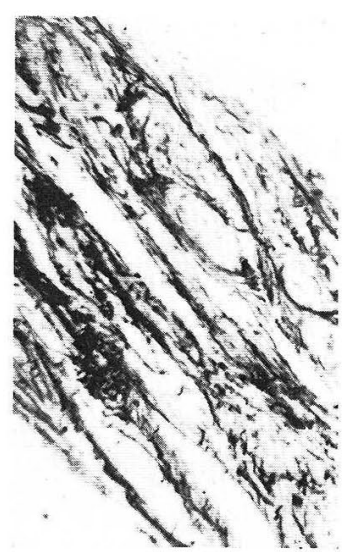

24 
Abb. 25. Längsschnitt des Centralteiles des Ligamentums. Aus einer 6 mal Gebärenden von 53 Jahren. Färbung mit Hämatoxylin und Eosin. $110 \times$

Abb. 26. Übersicht über den Querschnitt des Ligamentums. Aus einer 4mal Gebärenden von 50 Jahren. Färbung mit Resorcinfuchsin. $22 \times$

Abb. 27. Längsschnitt des Randteiles des Ligamentums. Aus einer 6 mal Gebärenden von 53 Jahren. Färbung mit Resorcinfuchsin. $110 \times$

Abb. 28. Übersicht über den Querschnitt des Ligamentums. Aus einer 2mal Gebärenden von 71 Jahren. Färbung mit Hämatoxylin und Eosin. $22 \times$

Abb. 29. Längsschnitt des Centralteiles des Ligamentums in Abb. 28. $110 \times$

Abb. 30. Längsschnitt des Randteiles des Ligamentums. Aus einer 2mal Gebärenden von 71 Jahren. Färbung mit Resorcinfuchsin. $110 \times$ 
Y. TAKEBAYASHI (4)



25

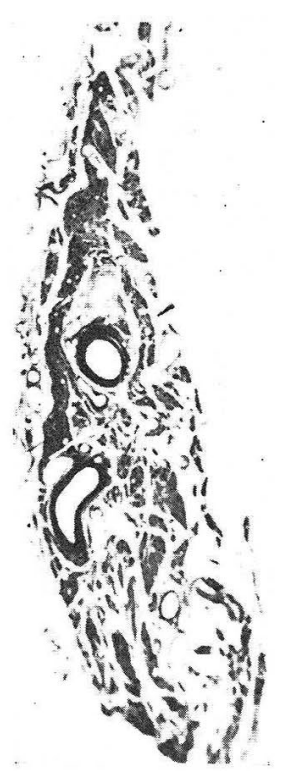

28

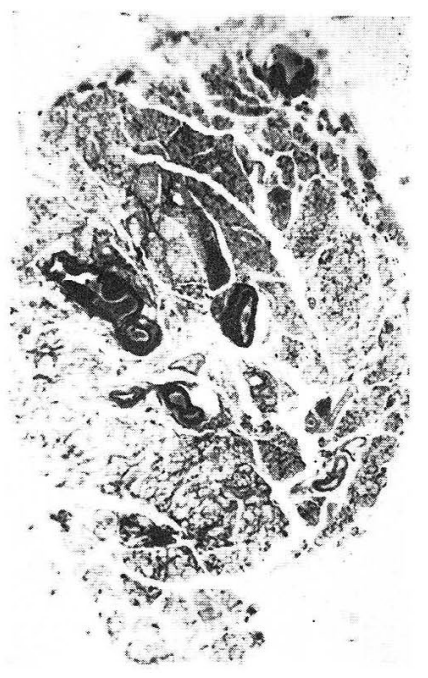

26
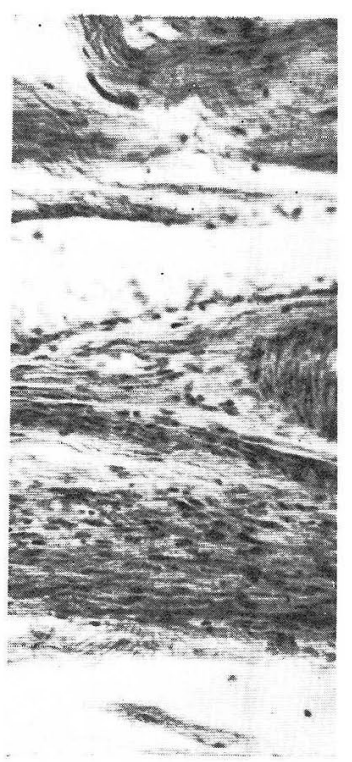

29
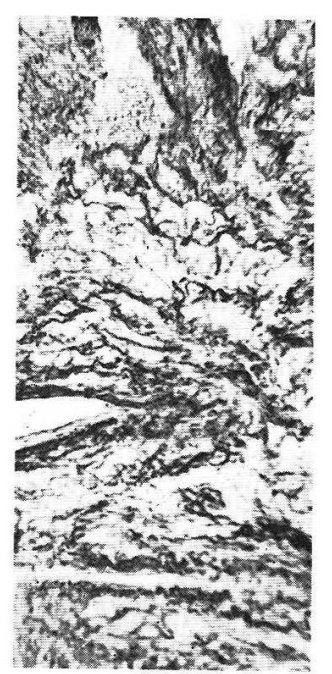

27

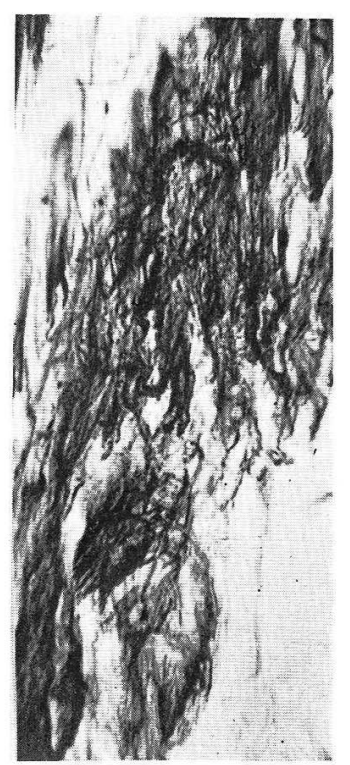

30 